\title{
Correlation between Atomic Structure and Superglide of an Incommensurate Grain Boundary in Au
}

\author{
U. Dahmen* T. Radetic*, J. Ye*, A. Minor*, D. Caliste and F. Lancon** \\ * National Center for Electron Microscopy, Materials Sciences Division, LBNL, Berkeley, CA \\ 94720 \\ ** Laboratoire de Simulation Atomistique (L_Sim), SP2M, INAC, CEA, 38054 Grenoble, France
}

Grain boundaries play a central role in the deformation of materials, especially in nanocrystalline materials where normal dislocation mechanisms are inhibited by the small grain size. In this work, we are investigating the behavior of an incommensurate grain boundary in gold by atomic resolution imaging, atomistic simulation and in-situ deformation experiments [1]. Simulations predict certain incommensurate boundaries to deform by a process of frictionless gliding, or superglide. To test this prediction experimentally, we used a bicrystal with a misorientation of $90^{\circ}$ around a common $<110>$ axis. In cubic materials, such a bicrystal makes a good model for incommensurate boundaries because at the grain boundaries $\{\mathrm{h} \mathrm{h} \mathrm{k}\}$ crystal planes of one grain align with $\{\mathrm{k} \mathrm{k} 2 \mathrm{~h}\}$ planes in the other grain. The ratio of plane spacings $\left(\sqrt{k^{2}+k^{2}+4 h^{2}} / \sqrt{h^{2}+h^{2}+k^{2}}=\sqrt{2}\right)$ is irrational and hence incommensurate. Figure 1 shows a high resolution micrograph of such a $\sqrt{ } 2$ incommensurate boundary where (200) planes of the lower grain are aligned with (110) planes of the upper grain. The structure of this boundary was computed by EAM calculations and tested against experimental images using image simulation, template matching and pattern recognition techniques. At the intersection with free surfaces, this boundary decomposed into a stable triangular Chevron-shaped defect about $1-2 \mathrm{~nm}$ in size, whose existence range depended on the orientation of the free surface relative to the grain boundary. For the surface orientation in Fig. 1, this instability is seen as a kink or facet in the last nanometer of the boundary.

For experimental tests of the mechanical behavior of this grain boundary we used a FIB machining to produce nanopillars from mazed bicrystal thin films of Au formed by vapor deposition on a single crystal Ge substrate. Figure 2 shows such nanopillars during FIB machining from the bicrystal thin film (left) and before and after in-situ compression with a nanoindenter. The grain boundary gliding during compression is readily apparent. The computed atomic structure and nanopillar model used for simulations are shown in Figure 3. The associated graph of force-displacement data compares the experimentally measured behavior (lower curve) with the simulated behavior under different conditions of temperature and boundary length. From this comparison between experimental and theoretical data, we conclude that the boundary deforms by superglide and that the main resistance to glide is due to relaxation at the free surfaces, which act as defects in the quasiperiodic structure of the infinite boundary.

\section{References}

[1] F. Lançon et al., Nano Letters (2010) (online DOI: 10.1021/n1903885).

[2] T. Radetic et al., Physical Review Letters 89 (2002) 085502.

[3] F. Lancon et al., Physical Review B 69 (2004) 172102.

[4] This work is supported by the Director, Office of Science, Office of Basic Energy Sciences, of the U.S. Department of Energy under Contract No. DE-ACO3-76SFOOO98, and by the Direction des Sciences de la Matière of the CEA. 


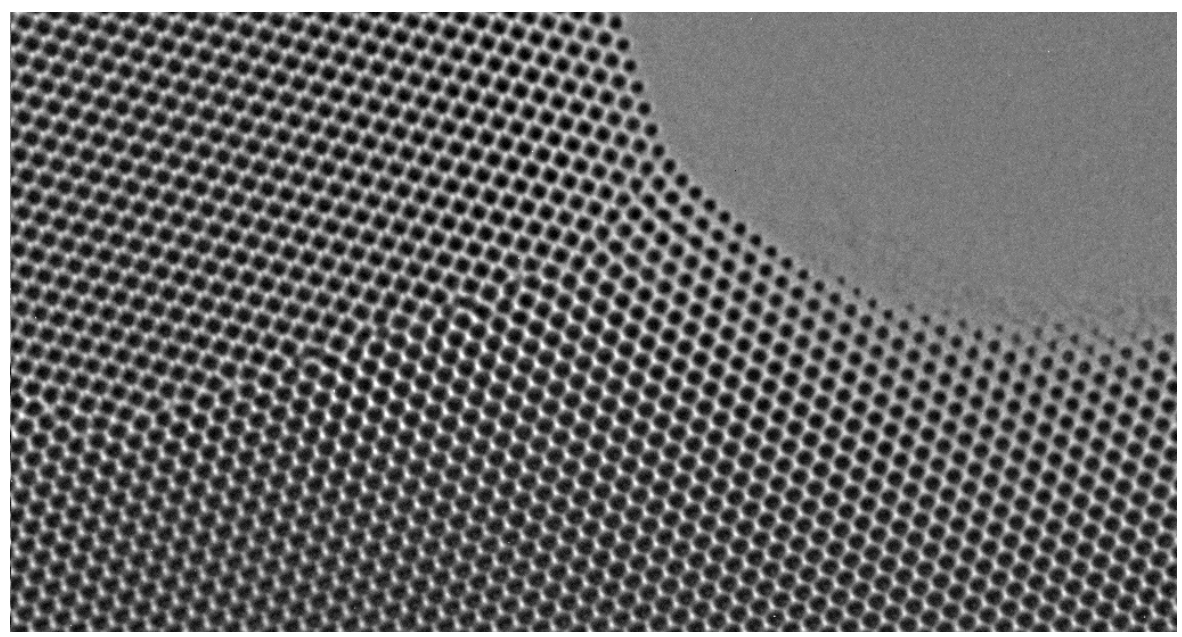

FIG. 1. Atomic resolution image of incommensurate $90^{\circ}<110>$ tilt grain boundary in mazed bicrystal of Au grown on single crystal Ge substrate.
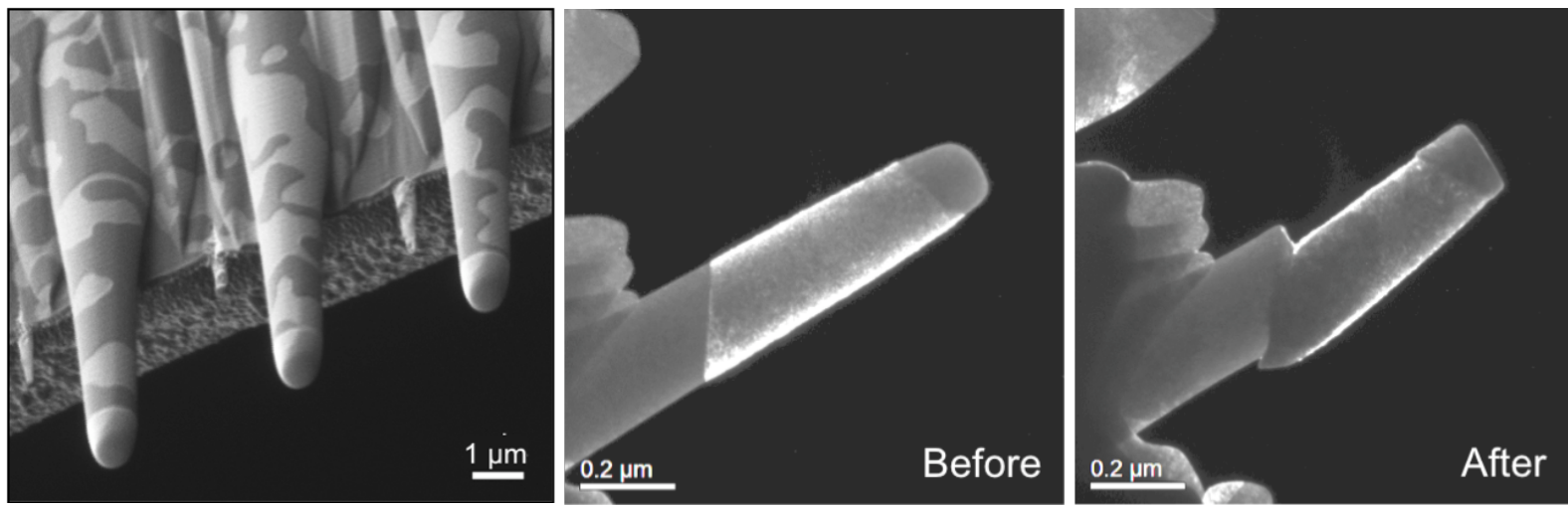

FIG. 2. Bicrystalline nanopillars for in-situ compression tests. Intermediate stage of pillar formation during FIB machining (left). Nanopillar with incommensurate grain boundary shown before and after in-situ compression with nanoindenter.
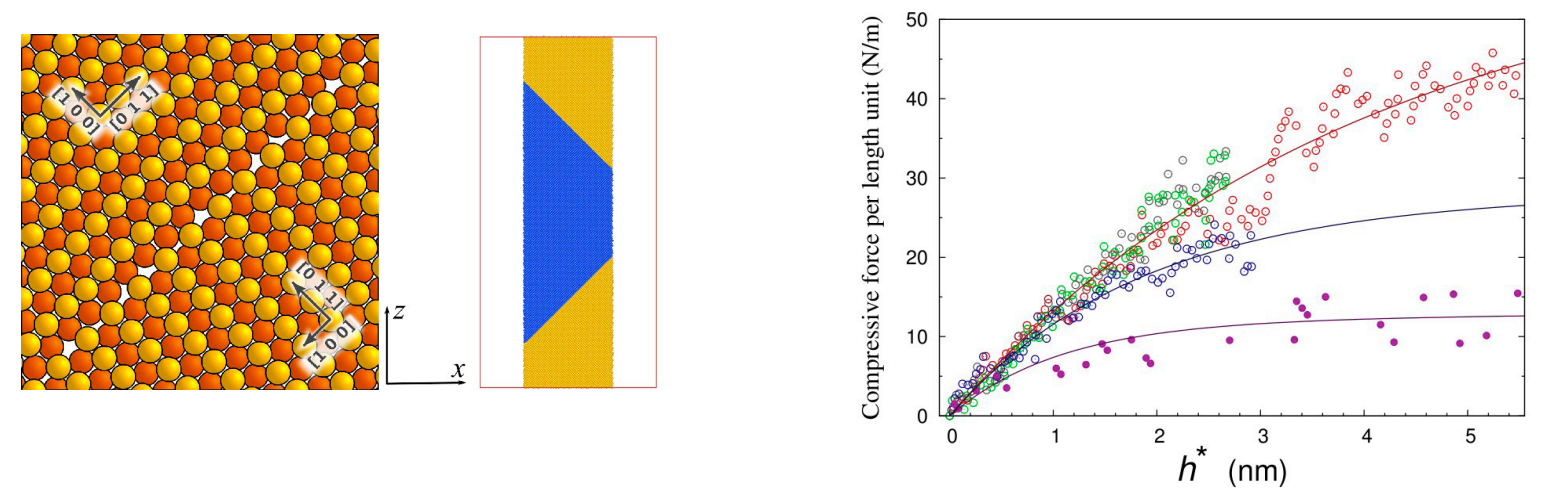

FIG. 3. Computed atomic structure of the boundary, and model geometry used to calculate forces during pillar compression (left). The graph compares experimental (lower curve) with simulated force-displacement behavior using the geometry on the left. Simulations at $0 \mathrm{~K}$ all superimpose on the same (upper) curve, regardless of boundary length or step structure. Center curve shows simulation at $300 \mathrm{~K}[1]$. 